\title{
Resultados al año de la rehabilitación tras fractura de fémur proximal en mayores de 84 años
}

\author{
J. J. BAZTÁN, M. FERNÁNDEZ-ALONSO, R. AGUADO, A. SOCORRO \\ Servicio de Geriatría. Hospital Central Cruz Roja. Madrid
}

OUTCOMES AFTER A YEAR OF REHABILITATION OF PROXIMAL
FEMUR FRACTURE IN OLDER THAN 84 YEARS

\section{RESUMEN}

Objetivo: Valorar los resultados de la rehabilitación en pacientes de 85 y más años con fractura de cadera y su persistencia al año, en relación con ancianos más jóvenes.

Método: Estudio prospectivo de todos los pacientes ingresados con fractura de cadera para recuperación funcional en una unidad geriátrica de media estancia entre mayo de 2000 y septiembre de 2002. Se recogieron variables clínicas (tipo de fractura, albúmina sérica e índice de comorbilidad de Charlson), situación funcional (Índice de Barthel), capacidad de deambulación (escala física de Cruz Roja) y la situación cognitiva (test de Pfeiffer). La situación funcional, deambulación e institucionalización fueron las variables de resultado al alta. El seguimiento se realizó telefónicamente recogiendo al año la incidencia de muerte, institucionalización, situación funcional y capacidad de deambulación.

Resultados: Se estudiaron 116 pacientes ( $42 \% \geq 85$ años; $85 \%$ mujeres). Tras el análisis logístico multivariante la edad $\geq 85$ no fue un factor asociado a dependencia funcional o institucionalización al alta. Tampoco se asoció al riesgo de muerte o institucionalización ni de deambulación dependiente al año del alta. Por contra, la presencia de deterioro cognitivo al ingreso y de deterioro funcional previo a la fractura se asociaron de forma independiente a la presencia de estos pobres resultados al alta y al año.

Conclusiones: La edad muy avanzada no aparece como un factor de riesgo independiente de pobres resultados tras la rehabilitación de fractura de cadera cuando se consideran otros factores clínicos, funcionales y mentales asociados.

PALABRAS CLAVE: Fractura de cadera. Anciano frágil. Rehabilitación. Hospital. Incapacidad. Mortalidad.

\begin{abstract}
Objective: To evaluate rehabilitation outcomes at discharge and at year patients older 85 years admitted to functional treatment after hip fracture.

Method: A prospective study of inpatients admitted after hip fracture to medium-stay geriatric unit between May 2000 and September 2002. Baseline, clinical variables (location of fracture, serum albumin and Charlson comorbidity index), functional status assessed by Barthel Index, mobility by Physical Red Cross Scale and cognitive status by Pfeiffer questionnaire. Functional status, mobility, institutionalization and death was evaluated at discharge and by phone interview at years after discharge.

Results: 116 patients were studied (42\% with 85 years and older, $85 \%$ women). After multivariable logistict analysis, age older than 84 years did not appear as a factor related to functional dependence or instituzionalitation at discharge or one year after. Indeed, oldest old age was not related with death or institutionalization and mobility dependence one year after. In the other hand, the presence of cognitive impairment at admission and functional impairment before hip fracture were independently variable associated to the presence of poor outcome at discharge and one year after.

Conclusions: Oldest old age does not appear as a independent risk factor of poor outcomes after rehabilitation ofhip fracture when other relatd clnical, functonal and mental factors are considered.
\end{abstract}

KEY WORDS: Hip fractures. Frail elderly. Rehabilitation outcome. Hospital. Disability. Mortality.

Baztán JJ, Fernández-Alonso M, Aguado R, Socorro A. Resultados al año de la rehabilitación tras fractura de fémur proximal en mayores de 84 años. An Med Interna (Madrid) 2004; 21: 433-440.

\section{INTRODUCCIÓN}

La fractura de cadera es una patología frecuente en la población anciana, con una incidencia de 517 casos por cada 100.000 personas mayores de 65 años, incidencia que aumenta exponencialmente con la edad (1). Las consecuencias de esta lesión conllevan un riesgo aumentado de incapacidad funcional, institucionalización y mortalidad a medio plazo. Es la quinta causa de desarrollo de incapacidad subaguda en varones mayores de 65 años, tras ictus, insuficiencia cardiaca, cáncer y neumonía, y la primera entre las mujeres (2). Presentan un ratio de mortalidad a los 6-12 meses que oscila entre el 20-35\% (3-6) y una incidencia de institucionalización añadida al año entre el 12-20\% $(5,6)$.

Trabajo aceptado: 1 de abril de 2004 
El progresivo abordaje interdisciplinario en la fase pre y posoperatoria ha contribuido a mejorar los resultados de morbi-mortalidad de estos pacientes (7-9). Sin embargo, hasta una tercera parte de los pacientes al alta de la planta de traumatología pueden ser subsidiarios de continuar su recuperación en unidades geriátricas de media estancia con objeto de reducir su incapacidad funcional $\mathrm{y}$, secundariamente, favorecer la permanencia en su domicilio previo (9).

El progresivo envejecimiento de la población, que en 2025 llegará al 22\% (un 12\% de los mismos mayores de 85 años) (10) hace prever un aumento progresivo de esta patología y sus consecuencias, por lo que mayores esfuerzos deben realizarse tanto en la prevención de fracturas de cadera como en la reducción de la incidencia de las secuelas anteriormente descritas a través de programas de rehabilitación. Con frecuencia la edad muy avanzada junto con el deterioro funcional previo y la presencia de deterioro cognitivo se han presentado como un factor de mal pronóstico de recuperación funcional, institucionalización y mortalidad (4,11-13).

El objetivo de nuestro estudio es conocer la respuesta a la rehabilitación intrahospitalaria de pacientes muy ancianos con fractura de fémur proximal, así como la influencia de la edad junto con otros factores clínicos, funcionales y mentales en los resultados de dicha rehabilitación al alta hospitalaria y un año después.

\section{PACIENTES Y MÉTODOS}

Se estudiaron todos los pacientes ingresados para recuperación funcional por fractura de fémur proximal en la Unidad Geriátrica de Media Estancia del Hospital Central de Cruz Roja y que cumplieron su ciclo de rehabilitación entre Mayo de 2000 y Septiembre de 2002. Dicha unidad consta de 30 camas y atiende a pacientes geriátricos que han sufrido deterioro funcional como consecuencia de diferentes patologías, que son atendidos por un equipo geriátrico que trabaja de forma interdisciplinaria en estrecha colaboración con el servicio de rehabilitación.

Los pacientes fueron distribuidos en dos grupos de edad: menores de 85 años y $\geq$ de 85 años. Se recogieron variables clínicas al ingreso (tipo de fractura, comorbilidad medida por el Índice de Charlson (14), nivel de albúmina sérica), situación funcional previa y al ingreso medida por el Índice de Barthel modificado por Shah et al $(15,16)$ (que recoge el grado de independencia en 10 actividades básicas de la vida diaria y que puntúa de 0 a 100, correspondiendo una puntuación $<60$ a dependencia moderada-severa, 60-89 dependencia leve $\mathrm{y} \geq 90$ a independencia), grado de movilidad evaluada por la Escala Física de Cruz Roja (17) (que gradúa la movilidad de 0 a 5, correspondiendo una puntuación de 0-2 a marcha independiente, 3 si requiere ayuda de una persona y 4-5 si requiere ayuda de dos personas para caminar o no camina), situación cognitiva al ingreso evaluada por el test de Pfeiffer (18) (que consta de 10 items, con un punto de corte para deterioro cognitivo si $\geq 5$ ), y situación social medida por el tipo de convivencia.

Al alta se recogió la situación funcional, grado de movilidad, días de estancia e incidencia de institucionalización. Como parámetros de resultados de rehabilitación se recogió la ganancia funcional durante el ingreso (Índice de Barthel al alta - Índice de Barthel al ingreso), considerando una ganan- cia $\geq 20$ como punto de corte de mejoría funcional clínicamente significativa. Igualmente se calculó la eficacia de la rehabilitación mediante el índice de Montebello, que calcula el porcentaje de pérdida funcional recuperado al alta respecto al sufrido tras la fractura de cadera al ingreso en la unidad y que viene determinado por la relación entre:

(I. Barthel alta - I. Barthel ingreso) / (I. Barthel previo - I. Barthel ingreso).

Una puntuación $>0,5$ ha sido presentada como indicador de resultado satisfactorio del tratamiento rehabilitador $(19,20)$.

Al alta los pacientes ingresados entre octubre de 2000 y septiembre de 2002 fueron seguidos telefónicamente a los 6 y 12 meses, recogiendo la incidencia de mortalidad e institucionalización, así como la situación funcional en actividades de la vida diaria y grado de movilidad.

Las variables cualitativas se expresaron en porcentajes, utilizando el test exacto de Fisher para su comparación entre grupos. Las variables cuantitativas se expresaron en medias \pm desviación estándar, utilizando el test de Wilcoxon para su comparación. Se consideró significación estadística si p<0,05.

Para estudiar la variables asociadas a muerte o institucionalización y deambulación independiente a los 12 meses se realizó un análisis logístico multivariante, en el que se incluyeron todas las variables basales consideradas.

El análisis se realizó en el paquete estadístico SPSS 9.0.

\section{RESULTADOS}

Durante el periodo del estudio ingresaron en la unidad 130 pacientes con fractura de fémur proximal. De ellos 14 no cumplieron el programa de rehabilitación y fueron excluidos del estudio (11 fueron reingresados en unidades de agudos, 3 de ellos por complicaciones de la osteosíntesis, 1 falleció en la unidad y 2 fueron dados de alta antes de 5 días desde el ingreso). De los 116 pacientes restantes se recogieron variables al ingreso y al alta.

En la tabla I se describen las características al ingreso de los pacientes de 85 y más años respecto a los menos ancianos. Destaca en los muy ancianos una mayor repercusión de la lesión, manifestada por una mayor incapacidad al ingreso en actividades de la vida diaria, dificultad para la deambulación, así como una mayor incidencia de deterioro cognitivo e hipoalbuminemia, siendo más frecuente la convivencia previa con otros familiares diferentes al cónyuge.

Los resultados funcionales del tratamiento en la unidad recogidos al alta se presentan en la tabla II. Si bien la magnitud de la mejoría obtenida es similar en ambos grupos de edad, el grupo de más ancianos presenta más frecuentemente al alta una peor situación funcional en actividades de la vida diaria y deambulación, diferencias que se mantienen en la evaluación al año del alta.

Sin embargo, cuando se analizaron conjuntamente las variables previas y al ingreso asociadas con peores resultados al alta de la unidad, no se encontró asociación entre la edad y estos peores resultados (salvo para la capacidad de deambulación independiente), siendo la peor situación funcional previa a la fractura (medida por el Índice de Barthel < 90) y la presencia de deterioro cognitivo al ingreso los principales determinantes de estos peores resultados (Tabla III). La asociación de Índice de Barthel al alta $<60$ e institucionalización como 
TABLA I

CARACTERÍSTICAS BASALES DE LOS PACIENTES

\begin{tabular}{|c|c|c|c|}
\hline Variables & $<84$ años & $\geq 85$ años & $p$ \\
\hline $\mathrm{n}$ & 67 & 49 & \\
\hline Edad (años) & $77,64( \pm 4,88)$ & $88,31( \pm 2,99)$ & $<0,01$ \\
\hline Mujeres & $76,1 \%$ & 91,85 & $<0,05$ \\
\hline $\begin{array}{l}\text { Tipo de fractura: } \\
\text { - Intracapsular (subcapital) } \\
\text { - Extracapsular: } \\
\text { - Basicervical } \\
\text { - Pertrocantérea } \\
\text { - Subtrocantérea }\end{array}$ & $\begin{array}{c}31,3 \% \\
7,5 \% \\
50,7 \% \\
9 \%\end{array}$ & $\begin{array}{l}50 \% \\
6,3 \% \\
37,5 \\
6,3 \%\end{array}$ & NS \\
\hline $\begin{array}{l}\text { Intervención quirúrgica: } \\
\text { - Prótesis parcial } \\
\text { - Clavo-placa (DHS) } \\
\text { - Otros } \\
\text { - No cirugía }\end{array}$ & $\begin{array}{c}33,3 \% \\
51,5 \% \\
12,1 \% \\
3 \%\end{array}$ & $\begin{array}{c}43,8 \% \\
33,3 \% \\
20,8 \% \\
2,1 \%\end{array}$ & NS \\
\hline I. Barthel previo & $85,27( \pm 19,08)$ & $81,61( \pm 19,15)$ & NS \\
\hline I. Barthel previo $<90$ & $39,4 \%$ & $55,1 \%$ & NS \\
\hline Deambulación previa independiente (1) & $78,8 \%$ & $79,8 \%$ & NS \\
\hline $\begin{array}{l}\text { Convivencia previa: } \\
\text { - Solos } \\
\text { - Cónyuge } \\
\text { - Familia } \\
\text { - Residencia }\end{array}$ & $\begin{array}{c}24 \% \\
38,8 \% \\
32,8 \% \\
1,5 \%\end{array}$ & $\begin{array}{c}16,3 \% \\
18,4 \% \\
59 \% \\
6 \%\end{array}$ & $<0,05$ \\
\hline I. Barthel al ingreso & $36,97( \pm 20,98)$ & $27,96( \pm 17,84)$ & $<0,05$ \\
\hline $\begin{array}{l}\text { Deambulación al ingreso }(1) \text { : } \\
\text { - Independiente } \\
\text { - Ayuda de } 1 \text { persona } \\
\text { - Ayuda } 2 \text { personas o no deambulan }\end{array}$ & $\begin{array}{c}4,5 \% \\
31,8 \% \\
63,6 \%\end{array}$ & $\begin{array}{c}0 \\
20,4 \% \\
79,6 \%\end{array}$ & 0,05 \\
\hline Deterioro cognitivo al ingreso (2) & $19,7 \%$ & $46,9 \%$ & $<0,01$ \\
\hline I. comorbilidad Charlson & $1,76( \pm 1,52)$ & $1,76( \pm 1,20)$ & NS \\
\hline Albúmina $<3,5 \mathrm{~g} / \mathrm{dl}$ & $42,4 \%$ & $16,3 \%$ & $<0,01$ \\
\hline
\end{tabular}

(1) Evaluada por la Escala Física de Cruz Roja (0-2: independiente; 3: ayuda de 1 persona; 4-5: ayuda de 2 personas o no deambulación)

(2) Evaluado por test de Pfeiffer $\geq 5$

variable conjunta de resultado al alta, tiene sentido desde el punto de vista asistencial, dado que una puntuación en el Índice de Barthel al alta $\geq 60$ es frecuentemente presentado como objetivo del ingreso, dado que se corresponde con una situación funcional suficiente para poder volver al domicilio.

Pese a esta asociación negativa, los pacientes con dependencia en actividades de la vida diaria previa a la fractura obtenían una recuperación del 54\% ( \pm 32 ) de la pérdida funcional sufrida al ingreso (medida por el Índice de Montebe1lo). De forma añadida, los pacientes con deterioro cognitivo al ingreso recuperaban en un 55\% $( \pm 48)$ del deterioro funcional sufrido al ingreso, y al alta el $14 \%$ eran capaces de deambular de forma independiente y un 58,3\% más caminaban con ayuda de una persona.

De los 116 pacientes evaluados al ingreso y al alta, se siguieron telefónicamente durante el año posterior 102 pacientes (todos aquellos ingresados consecutivamente desde octubre de 2000 y septiembre de 2002). No hubo diferencias entre los pacientes seguidos y no seguidos en edad, tipo de fractura, situación funcional y grado de deambulación previa al ingreso y al alta y estado cognitivo al ingreso. De estos pacientes en 5 no se pudieron obtener datos fiables a los 12 meses, por lo que finalmente fueron evaluados 97. La evolución funcional, incidencia de muerte e institucionalización en el seguimiento se presenta en los gráficos 1 y 2 .

El análisis bivariante entre variables basales y los resultados de muerte, institucionalización y deambulación dependiente de otra persona al año del alta se presenta en la tabla IV. Para realizar el análisis multivariante se decidió asociar los resultados de muerte e institucionalización al año, dado el escaso número de eventos registrado para el análisis por separado (Tabla V). Al igual que en el análisis realizado al alta, la dependencia funcio- 
TABLA II

RESULTADOS DE LOS PACIENTES AL ALTA DE LA UNIDAD Y AL AÑO DEL ALTA

\begin{tabular}{|c|c|c|c|}
\hline Variables & $<84$ años & $\geq 85$ años & $p$ \\
\hline$n$ & 67 & 49 & \\
\hline I. Barthel (IB) al Alta & $65,77( \pm 24,51)$ & $57,14( \pm 17,99)$ & $<0,05$ \\
\hline I. Barthel alta $>60$ puntos & $69,7 \%$ & $49 \%$ & $<0,05$ \\
\hline $\begin{array}{l}\text { Ganancia Funcional } \\
\text { (IB alta-IB ingreso) }\end{array}$ & $28,80( \pm 18,54)$ & $29,18( \pm 15,14)$ & NS \\
\hline $\begin{array}{l}\text { I. Barthel alta-I. Barthel ingreso } \\
>20 \text { puntos }\end{array}$ & $75,8 \%$ & $73,5 \%$ & NS \\
\hline Indice de Montebello & $0,63( \pm 0,41)$ & $0,62( \pm 0,42)$ & NS \\
\hline I. Montebello >0,5 & $73,1 \%$ & $61,2 \%$ & NS \\
\hline $\begin{array}{l}\text { Deambulación al alta (1): } \\
\text { - Independiente } \\
\text { - Ayuda de } 1 \text { persona } \\
\text { - Ayuda } 2 \text { personas o } \\
\quad \text { no deambulan }\end{array}$ & $\begin{array}{c}53 \% \\
30,3 \% \\
16,7 \%\end{array}$ & $\begin{array}{l}26,5 \% \\
61,2 \% \\
12,2 \%\end{array}$ & $<0,01$ \\
\hline Estancia (días) & $21,67( \pm 8,60)$ & $26,49( \pm 11,08)$ & $<0,05$ \\
\hline Alta a Residencia & $11,9 \%$ & $18,4 \%$ & NS \\
\hline $\begin{array}{l}\text { Resultados a los } 12 \text { meses del alta (2): } \\
\text { - Muerte } \\
\text { - Institucionalización } \\
\text { - I. Barthel > } 60 \\
\text { - Deambulación (1): } \\
\text { - Independiente } \\
\text { - Ayuda de } 1 \text { persona } \\
\text { - Ayuda } 2 \text { personas o no deambulan }\end{array}$ & $\begin{array}{l}12,7 \% \\
18,5 \% \\
68,8 \% \\
62,5 \% \\
20,8 \% \\
16,7 \%\end{array}$ & $\begin{array}{c}16,7 \% \\
21 \% \\
47,1 \% \\
42,4 \% \\
42,4 \% \\
15,2 \%\end{array}$ & $\begin{array}{c}\text { NS } \\
\text { NS } \\
0,07 \\
<0,05\end{array}$ \\
\hline
\end{tabular}

${ }^{1}$ Evaluada por la Escala Física de Cruz Roja (0-2: independente; 3: ayuda de 1 persona; 4-5: ayuda de 2 personas o no deambulación). ${ }^{2}$ Los porcentajes situación funcional y deambulación corresponden a la población superviviente al año.

nal previa al ingreso y la presencia de deterioro cognitivo al ingreso eran las principales variables asociadas a peores resultados a los 12 meses del alta. Pese a este peor pronóstico, fueron capaces de deambular de forma independiente o con ayuda de una persona el $69 \%$ de supervivientes con dependencia funcional previa al ingreso y el $60 \%$ de pacientes con deterioro cognitivo al ingreso en la unidad (tabla IV).

Por último, en los pacientes supervivientes al año del alta se objetivó una pérdida funcional del $22 \%$ ( \pm 34 ) respecto a la situación previa a la fractura (medida por el Índice de Barthel) que supuso una mejoría significativa $(\mathrm{p}<0,01)$ respecto a la pérdida funcional objetivada al ingreso que fue del $60 \%$ $( \pm 0,19)$. En este sentido, el porcentaje de dicha pérdida al ingreso recuperado al alta $(65 \% \pm 0,58)$, se mantenía en estos pacientes a los 12 meses $(61 \% \pm 0,39)$.

\section{DISCUSIÓN}

Los datos presentados reflejan la respuesta al tratamiento multidisciplinario encaminado a la recuperación funcional de pacientes ancianos ingresados en una unidad geriátrica de media estancia tras sufrir una fractura de cadera. Los pacientes estudiados fueron seleccionados previamente a su ingreso en función de la necesidad de recuperación funcional y la presencia de un objetivo concreto de mejoría (que no necesariamente era la recuperación completa de la situación funcional previa) encaminado a facilitar la reintegración en su domicilio previo. En este sentido, los resultados obtenidos no son extrapolables al conjunto de toda la población de pacientes con fractura de cadera. Sin embargo, dichos resultados pueden ayudar a conocer la evolución y necesidad de tratamiento rehabilitador de aquellos pacientes que tras reparación quirúrgica de una fractura de cadera no consiguen una recuperación funcional suficiente para volver a su domicilio previo, y que en algunos estudios se ha cifrado en un 34\% (9). Este objetivo explicaría que si bien entre un 20-30\% de pacientes ingresados en unidades de cirugía ortopédica y traumatología (COT) por fractura de cadera viven previamente en residencias $(4,9)$, solo el 3,4\% de los pacientes de este estudio tenían esta ubicación previa.

La población estudiada se caracteriza por presentar una edad muy avanzada (algo por otra parte muy lógico cuando hablamos de fracturas de cadera) y elevada incidencia de dete- 
TABLA III

ANÁLISIS MULTIVARIANTE DE REGRESIÓN LOGÍSTICA DE VARIABLES AL INGRESO ASOCIADAS CON "POBRES RESULTADOS" AL ALTA (RESULTADOS EXPRESADOS MEDIANTE "ODDS RATIO" CON INTERVALO DE CONFIANZA AL 95\%) ( $n=114$ )

\begin{tabular}{|c|c|c|c|}
\hline Variable & o deambulación independiente (1) & I. Montebello $<0,5$ & 1. Barthel <60 (2) ó Residencia al alta \\
\hline Edad $\geq 85$ años & $2,67(0,98-7,31)$ & $1,12(0,41-3,02)$ & $1,14(0,45-2,86)$ \\
\hline Sexo femenino & $1,16(0,32-4,24)$ & $4,06(0,77-21,50)$ & $2,96(0,71-12,38)$ \\
\hline I. Barthel previo <90 (2) & $4,50(1,68-12,04)$ & $0,26(0,08-0,83)$ & $3,98(1,62-9,79)$ \\
\hline Convivencia con pareja & $0,81(0,27-2,50)$ & $1,34(0,41-4,32)$ & $1,21(0,40-3,66)$ \\
\hline Fractura extracapsular & $1,43(0,55-3,16)$ & $0,50(0,19-1,29)$ & $0,95(0,39-2,30)$ \\
\hline Deterioro cognitivo al ingreso (3) & $5,23(1,53-17,94)$ & $9,86(3,00-32,41)$ & $2,60(0,98-6,92)$ \\
\hline $\begin{array}{l}\text { I. comorbilidad Charlson: } \\
-0 \\
-1-2 \\
-\geq 3\end{array}$ & $\begin{array}{c}1 \\
1,32(0,40-4,39) \\
1,19(0,28-5,08)\end{array}$ & $\begin{array}{c}1 \\
0,61(0,18-1,98) \\
2,21(0,54-9,10)\end{array}$ & $\begin{array}{c}1 \\
0,81(0,26-2,52) \\
0,81(0,21-3,10)\end{array}$ \\
\hline Albúmina sérica $<3,5 \mathrm{~g} / \mathrm{dl}$ & $0,68(0,25-1,84)$ & $0,89(0,31-2,61)$ & $1,44(0,56-3,70)$ \\
\hline
\end{tabular}

${ }^{1}$ No deambulación independiente medida por la Escala Física de Cruz Roja (CRF) $\geq 3$.

${ }^{2}$ I. Barthel <60: dependencia moderada-severa en actividades básicas de la vida diaria; $\geq 90$ : independencia.

${ }^{3}$ Deterioro cognitivo evaluado por una puntuación en el Test de Pfeiffer $\geq 5$

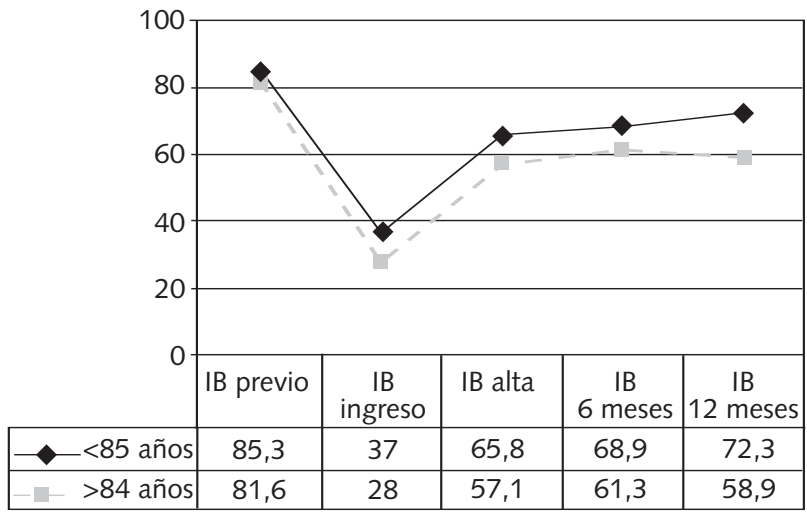

Fig. 1. Evolución funcional de la dependencia en actividades básicas de la vida diaria (medida por el Índice de Barthel) de pacientes con fracturas de cadera por grupos de edad.

rioro funcional previo y cognitivo al ingreso, factores todos ellos que pueden condicionar la ausencia de tratamiento rehabilitador posterior a la cirugía reparadora de la fractura por haber sido relacionados con mal pronóstico de recuperación funcional (4,11-13). Sin embargo, dadas las graves secuelas sobre el pronóstico y calidad de vida de estos pacientes, así como sobre la posterior necesidad de recursos asistenciales sanitarios y sociales, sería necesario profundizar sobre dichos factores pronósticos con objeto de individualizar en la detección y selección de aquellos pacientes que se beneficiarían de un tratamiento rehabilitador hospitalario y, de esta manera, optimizar los escasos recursos terapéuticos existentes en este sentido.

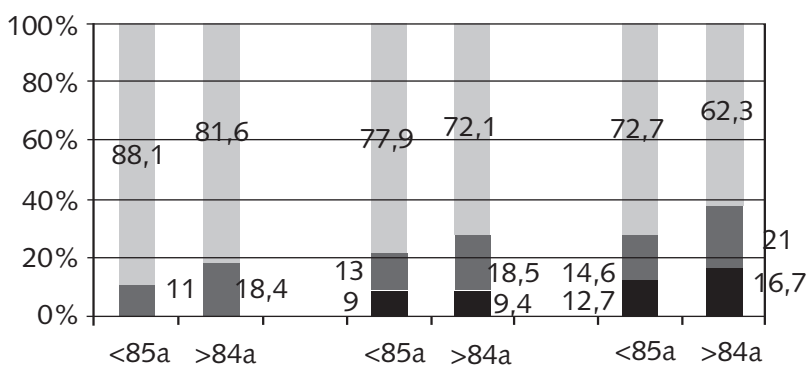

- Muerte Institucionalización $\square$ Domicilio
Fig. 2. Incidencia de muerte e institucionalización por grupos de edad.

La edad avanzada ha sido referida como un factor de riesgo de peor situación funcional tras rehabilitación de fractura de cadera (21). En nuestro estudio, los pacientes más ancianos presentaron también una peor situación funcional y menor capacidad de deambulación al alta y al año en relación con los más jóvenes, aunque el beneficio obtenido del tratamiento rehabilitador fue similar en ambos grupos. Sin embargo la edad no fue por si sola un factor de riesgo independiente de dicha peor situación funcional en el análisis multivariante. Probablemente, la mayor frecuencia de deterioro funcional previo a la fractura, junto con la mayor incidencia de deterioro cognitivo al ingreso asociada a la edad son factores de confusión que contribuyen a magnificar la importancia de la edad 
TABLA IV

ANÁLISIS BIVARIANTE ENTRE VARIABLES BASALES Y AL ALTA Y RESULTADOS AL AÑO

\begin{tabular}{lcccc}
\hline Variable & $n$ & Muerte & Institucionalización & No deambulación independiente(1) \\
\hline Eventos/pacientes & & $14 / 97(14,4 \%)$ & $15 / 81(18,5 \%)$ & $37 / 83(45,7 \%)$
\end{tabular}

Edad:

- $<85$ años

55

12,7

16,7

37,5

$-\geq 85$ años

42

16,7

21,2

57,6

Sexo:

- varón

17

$29,4^{*}$

25

17,4

25

- mujer

80

11,3

49,3

Convivencia previa:

- Solo

21

9,5

12,9

26,3

$7,4^{*}$

33,3

- Cónyuge

16,7

22,9

33,3

42

I. Barthel previo (2):

$-\geq 90$

$-<90$

$-\mathrm{NO}$

- SI

I. Comorbilidad de Charlson:

$-0$

$-1-2$

$-\geq 3$

Albúmina sérica $(\mathrm{g} / \mathrm{dl})$ :

$$
-\geq 3,5
$$

$-<3,5$

I. Barthel al alta (2):

$-\geq 60$

****

- <40

Tipo de fractura:

- intracapsular

- extracapsular
50

42,6

Los datos están expresados en porcentajes.

Comparaciones realizadas con la prueba exacta de Fisher: * $p<0,10 ;{ }^{*} p<0,05 ; * * p<0,01$. **** $p<0,05$ evaluada por "chi" cuadrado de tendencias

(1) No deambulación independiente medida por la Escala Física de Cruz Roja (CRF) $\geq 3$.

(2) I.Barthel categorizado por grado de dependencia en: independiente $(\geq 90)$; dependencia leve $(60-89)$; moderada $(40-59)$ y severa $(<40)$

(3) Deterioro cognitivo evaluado por Test de Pfeiffer al ingreso (NO-<5; $\mathrm{SI}-\geq 5$ ).

cronológica como factor de riesgo independiente. Este hecho reflejaría la necesidad de contemplar otros factores diferentes a la edad para seleccionar pacientes que potencialmente se beneficiarían de la prolongación del tratamiento rehabilitador intrahospitalario en unidades especializadas.

Los resultados de nuestro estudio son congruentes con la literatura en detectar la presencia de deterioro cognitivo como el principal factor riesgo independiente tanto de mortalidad, institucionalización como de peor situación funcional en pacientes con fractura de cadera $(4,5,12,13,19)$. Sin embargo, la presencia de deterioro cognitivo no debiera ser sinónimo de ausencia de beneficio al tratamiento rehabilitador intrahospitalario, ya que cerca del $70 \%$ recuperaban la capacidad de deambular al alta de forma independiente o con mínima ayuda de una persona, capacidad de deambulación que persistía al año en el $60 \%$. Recientemente se han comunicado datos en este sentido que objetivan una satisfactoria recuperación de la marcha tras un programa de rehabilitación adaptado a la capacidad de estos pacientes, especialmente en aquellos que presentan un grado de deterioro cognitivo leve-moderado $(19,22,23)$. Probablemente este grado favorable de respuesta a la rehabilitación está relacionado con la estabilidad de la osteosíntesis, el control de la confusión añadida y la menor complejidad del tratamiento rehabilitador, frente al requerido por pacientes con otras patologías como el ictus.

El deterioro funcional es un marcador de fragilidad y factor de riesgo de morbimortalidad más relevante que la edad cronológica en la población anciana independientemente de 
TABLA V

ANÁLISIS MULTIVARIANTE DE REGRESIÓN LOGÍSTICA DE VARIABLES AL INGRESO ASOCIADAS CON "POBRES RESULTADOS" A LOS 12 MESES DEL ALTA (RESULTADOS EXPRESADOS MEDIANTE "ODDS RATIO" CON INTERVALO DE CONFIANZA AL $95 \%)$

\begin{tabular}{lcc}
\hline Variable & Muerte o Institucionalización & No deambulación independiente (1) \\
\hline Edad $\geq 85$ años & $1,74(0,55-5,45)$ & $1,05(0,25-4,39)$ \\
Sexo femenino & $0,06(0,01-0,37)$ & $1,18(0,15-9,39)$ \\
I. Barthel previo $<90$ & $2,89(0,98-8,52)$ & $9,90(2,67-36,75)$ \\
Convivencia con pareja & $0,11(0,02-0,61)$ & $0,74(0,16-3,37)$ \\
Fractura extracapsular & $2,38(0,83-6,86)$ & $0,68(0,18-2,50)$ \\
Deterioro cognitivo al ingreso (2) & $2,80(0,94-8,39)$ & $20,26(3,31-124,11)$ \\
I. de comorbilidad de Charlson: & & 1 \\
-0 & 1 & $1,12(0,21-5,88)$ \\
$-1-2$ & $0,77(0,19-3,12)$ & $0,94(0,13-6,76)$ \\
$-\geq 3$ & $0,69(0,13-3,60)$ & $0,98(0,25-3,78)$ \\
Albúmina sérica $<3,5 \mathrm{~g} / \mathrm{dl}$ & $0,93(0,30-2,90)$ & \\
\hline
\end{tabular}

${ }^{1}$ No deambulación independiente medida por la Escala Física de Cruz Roja (CRF) $\geq 3$. 2Deterioro cognitivo evaluado por Test de Pfeiffer al ingreso (NO$<5 ; \mathrm{SI}-\geq 5)$

su patología subyacente (24). Es por ello que la detección de dependencia en actividades básicas de la vida diaria es también un factor asociado a peores resultados en ancianos con fractura de cadera $(4,5,12,13)$. Sin embargo, al igual que ocurre con la presencia de deterioro cognitivo, es un condicionante habitual en la práctica geriátrica especializada que contribuye no tanto a descartar los beneficios terapéuticos como a relativizar los objetivos del tratamiento rehabilitador. Así, al año del alta, si bien solo un $22 \%$ de los supevivientes caminaban de forma independiente, el hecho de que un $50 \%$ añadido caminaran con ayuda de una persona (frente al $87 \%$ que presentaban incapacidad para la deambulación o necesidad de ayuda de dos personas, al ingreso en la unidad) puede ser interpretado también como un beneficio relevante en tanto que reduce el grado de dependencia y necesidad de cuidados de estos pacientes que favorece su permanencia en el domicilio habitual.

Por último otros resultados encontrados son menos consistentes en la literatura. Así, si bien se ha relacionado en algunos estudios el sexo masculino con una mayor incidencia de mortalidad a corto y medio plazo tras fractura de cadera $(4,12)$, esto no ha sido confirmado en otros estudios. Algunos autores han relacionado este mayor riesgo de muerte con una mayor severidad en la caída que ocasionó la fractura, así como con una mayor comorbilidad o fragilidad previa, hechos que no podemos confirmar en nuestro estudio. Por otra parte, la relación inversa entre convivencia con pareja e institucionalización al año puede simplemente reflejar la menor tendencia al ingreso en residencia cuando existe un cuidador estable (cónyuge) o cuando se plantea la ubicación separada de uno de los miembros de la pareja.

\section{Bibliografía}

1. Serra JA, Garrido G, Vidán M, Marañón E, Brañas F, Ortiz J. Epidemiología de la fractura de cadera en ancianos en España. An Med Interna (Madrid) 2002; 19: 389-95.

2. Ferrucci L, Guralnik J, Pahor M, Conti MC, Havlik RJ. Hospital diagnoses, medical charges, and nursing home admissions in the year when older persons become severely disabled. JAMA 1997; 277: 728-34.

3. Sosa M, Segarra MC, Limiñana JM, Hernández D, González A, Betancor P. Morbilidad y mortalidad de la fractura osteoporótica de la extremidad proximal del fémur tras un año de seguimiento. Med Clin (Barc) 1993; 101: 481-3.

4. Pagés E, Cuxart A, Iborra J, Olona M, Bermejo B. Fracturas de cadera en el anciano determinantes de mortalidad y capacidad de marcha. Med Clin (Barc) 1998; 110: 687-91.

5. Parker MJ, Palmer CR. Prediction of rehabilitation after hip fracture. Age Ageing 1995; 24: 96-8.
6. Rosell PA, Parker MJ. Functional outcome after hip fracture. A 1-year prospective outcome study of 275 patients. Injury 2003; 34: 529-32.

7. Cameron I, Crotty M, Currie C, Finnegan T, Gillespie L, Handoll H et al. Geriatric rehabilitationfollowing fractures in older people: a systematic review. Health Technol Assess 2000; 4 (2): 1-83.

8. Gillespie J. Hip fracture. Br Med J 2001; 321: 968-75.

9. González-Montalvo JI, Alarcón T, Sáez P, Bárcena A, Gotor P, del Río M. La intervención geriátrica puede mejorar el curso clínico de los ancianos geriátricos con fractura de cadera. Med Clin (Barc) 2001; 116: 1-5.

10. Fernández Cordón JA. Proyecciones de la población española. Madrid: Instituto de Economía y Geografía. Consejo Superior de Investigaciones Científicas, 1998.

11. Marottoli RA, Berckman LF, Cooney LM. Decline in physical function following hip fracture. J Am Geriatr Soc 1992; 40: 861-6. 
12. Cree M, Soskolne CL, Belseck E, Hornig J, McElhaney JE, Brant R et al. Mortality and institutionalization following hip fracture. J Am Geriatr Soc 2000; 48: 283-8.

13. Adunsky A, Lusky A, Arad M, Heruti RJ. A comparative study of rehabilitation outcomes of elderly hip fractures patients: the adventage of a comprehensive orthogeriatric approach. J Gerontol 2003; 58A: 542-7.

14. Charlson ME, Pompei P, Ales KL, MacKenzie CR. A new method of classifying prognostic comorbidity in longitudinal studies: development and validation. J Chron Dis 1987; 40: 373-83.

15. Shah S, Vanclay F, Cooper B. Improving the sensitivity of the Barthel Index for stroke rehabilitation. J Clin Epidemiol 1989; 42: 703-9.

16. Cid-Ruzafa J, Damián-Moreno J. Valoración de la discapacidad física: el Indice de Barthel. Rev Esp Salud Pública 1997; 71: 127-37.

17. Regalado P, Valero C, González-Montalvo JI, Salgado A. las escalas de la Cruz Roja veinticinco años después: estudio de su validez en un servicio de geriatría. Rev Esp Geriatr Gerontol 1997; 32: 93-9.

18. Martínez de la Iglesia J, Dueñas R, Onís MC, Aguado C, Albet C, Luque R. Adaptación y validación al castellano del cuestionario de Pfeiffer (SPMSQ) para detectar la existencia de deterioro cognitivo en personas mayores de 65 años. Med Clin (Barc) 2001; 117: 129-34.

19. Heruti RJ, Lusky A, Barell V, Othy A, Adunsky A. Cognitive status at admission: does it affect the rehabilitation outcome of elderly patients with hip fracture?. Arch Phys Med Rehabil 1999; 80: 432-6.

20. Jones GR, Miller TA, Petrella RJ: evaluation of rehabilitation outcomes in older patients with hip fractures. Am J Phys Med Rehabil 2002; 81: 489-97.

21. Lieberman D, Lieberman D. Rehabilitation after proximal femur fracture surgery in the oldest old. Arch Phys Med Rehabil 2002; 83: 1360-3.

22. Goldstein F, Strasser DC, Woodard JL, Roberts VJ. Functional outcomes of cognitively impaired hip fracture patients on a geriatric rehabilitation unit. J Am Geriatr Soc 1997; 45: 35-42.

23. Huusko TM, Karppi P, Avikainen V, Kautiainen H, Sulkava R. Randomised, clinically controlled trial of intensive geriatric rehabilitation in patients with hip fracture: subgroup analysis of patients with dementia. Br Med J 2000; 321; 1107-11.

24. Baztán JJ, González-Montalvo JI, Solano JJ, Hornillos M. Atención sanitaria al anciano frágil: de la teoría a la evidencia científica. Med Clin (Barc) 2000; 115: 704-17. 